\title{
THE DIELECTRIC BREAKDOWN OF MAGNETIC FLUIDS
}

The development of dielectric breakdown and the DC dielectric breakdown voltage of magnetic fluids based on inhibited transformer oil ITO 100 were investigated in parallel orientations of external magnetic field. It was shown that the breakdown voltage is strongly influenced by the magnetic nanoparticles. The magnetic fluids with the volume concentration of $1 \%$ had better dielectric properties than pure transformer oil. The increase of breakdown voltage was interpreted on the base of the bubble theory of breakdown.

Keywords: transformer oil, magnetic fluid, relative permittivity, breakdown voltage

\section{Introduction}

Magnetic fluids based on inhibited transformer oil ITO 100 have been shown to provide thermal and dielectric benefits to the power transformers. They can improve transfer of heat by enhancing the fluid circulation within transformer windings [1], while also minimizing the effect of moisture on typical insulating fluids. Better dielectric properties of magnetic fluids than pure transformer oil and the use as high-voltage insulation was found for low volume concentration of $0.1 \%[2,3]$. Such transformer oil shows the strong dependence of dielectric breakdown strength on aggregation effects of magnetic nanoparticles [3].

A magnetic fluid is a colloidal suspension of nano-sized magnetic particles covered with a surfactant layer in a carried liquid. Particles of this size are monodomain and interaction between them may lead to their agglomeration and subsequent sedimentation. To avoid these undesired side effects magnetic particles can be coated with a surfactant that produces entropic repulsion. The macroscopic magnetic properties of the magnetic fluid are determined by the orientation of magnetic moments of nanoparticles in the external magnetic field. An externally applied magnetic field induces ordering of magnetic moments of the particles giving rise to magnetization of the sample as a whole and can cause certain amount of colloidal particles to join into clusters as long as hundreds of nanometers or more $[4,5]$.

The aim of the research reported in this article was to study the dielectric properties and DC dielectric breakdown voltage of magnetic fluids based on ITO 100 transformer oil. The effect of external magnetic field induced structuralization of magnetic particles in magnetic fluid on the dielectric properties were studied too.

\section{Experimental setup and results}

Fig. 1 shows the schematic diagram of the experimental setup, which includes HVdc power supply TESLA BS 221 (max voltage $10 \mathrm{kV}$ and current $3 \mathrm{~mA}$ ), electrode system, electric diagnostics and electromagnet. Sphere-to-sphere $\mathrm{Cu}$ electrodes with radius of $1 \mathrm{~cm}$ and plane-to-plane $\mathrm{Cu}$ electrodes with radius of $2.7 \mathrm{~cm}$ were used as the electrode system. The distance of electrodes was measured by metric gauge blocks with accuracy of $0.01 \mathrm{~mm}$. New and unfiltered transformer oil - ITO 100 was filled into a discharge chamber $\left(0.2 \mathrm{dm}^{3}\right)$ and electrodes were again cleaned after series of 7 breakdowns. The capacitor value of $1 \mathrm{nF}$ and resistance of $1 \mathrm{M} \Omega$ were chosen. The applied voltage and current were measured using a high voltage probe $(\mathrm{E} 253 / 01,10 \mathrm{MHz})$ and a Rogowski coil (Pearson Current monitor 110A, $10 \mathrm{kA}, 20 \mathrm{MHz}, 20 \mathrm{~ns}$ ). The signals were recorded on Agilent technologies DSO3202A, $200 \mathrm{MHz}$ digital oscilloscope. The time developments of resistance of magnetic fluids were measured using C.A 6549 Megaohmeter.

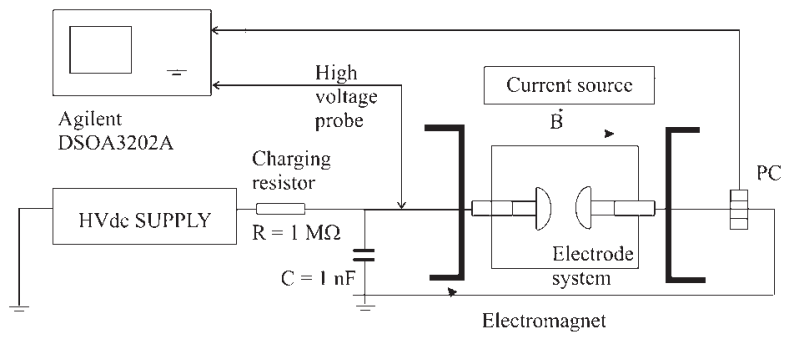

Fig. 1 The experimental setup

The magnetic fluids used in experiments consisted of magnetite particles $\left(\mathrm{FeO} \mathrm{Fe}_{2} \mathrm{O}_{3}\right)$, the mean diameter $D=10.6 \mathrm{~nm}$, coated

\footnotetext{
* Jozef Kudelcik ${ }^{1}$, Peter Bury ${ }^{1}$, Vlasta Zavisova ${ }^{2}$, Milan Timko ${ }^{2}$, Peter Kopcansky ${ }^{2}$

${ }^{1}$ Department of Physics, Faculty of Electrical Engineering, University of Zilina, Slovakia, E-mail: kudelcik@fyzika.uniza.sk

${ }^{2}$ Department of Magnetism, Institute of Experimental Physics SAS, Kosice, Slovakia
} 
with oleic acid as a surfactant, dispersed in inhibited ITO 100 transformer oil. The original magnetic fluid used in our experiment had density equal to $1.071 \mathrm{~g} / \mathrm{cm}^{3}$ and its saturation magnetization was $8.81 \mathrm{mT}$. From this magnetic fluid and pure ITO 100 transformer oil the magnetic fluids with volume fractions $0.2 \%, 1 \%$ and $2 \%$ were prepared. The acoustic velocity in these magnetic fluids without magnetic field is $c=1652 \mathrm{~m} / \mathrm{s}\left(25^{\circ} \mathrm{C}\right)$.

Each value of the breakdown voltage was measured seven times and the maximum and minimum values were omitted in the calculation of its mean value according to the rules of high voltage techniques [6]. Time intervals between breakdowns were 5 minutes. The experimental error of the breakdown voltage determination was $\pm 10 \%$

One of parameters that determine the quality of transformer oil is the isolation resistance. This parameter gives information on isolation stage which can be decreased by water content, solid impurities and gases dissolved in oil [7]. Transformer oil or magnetic fluids were in the electrode system with plane-to-plane $\mathrm{Cu}$ electrodes and the measurements were made at $500 \mathrm{~V} \mathrm{DC}$ by Megaohmeter. The isolation resistances were measured at following times: 15, 30, 40 and 60 seconds. From measured values the polarization index $p$ calculated as: $p=R_{60} / R_{15}$, which is also important in transformers diagnostics, can be determined. Values of insulation resistance and polarization index observed for various times are in Table 1 .

The resistance of transformer oil and magnetic

Table 1 fluids summarized for various times.

\begin{tabular}{|c|c|c|c|c|c|}
\hline Liquid & $R_{15}[\mathrm{G} \Omega]$ & $R_{30}[\mathrm{G} \Omega]$ & $R_{40}[\mathrm{G} \Omega]$ & $R_{60}[\mathrm{G} \Omega]$ & $p$ \\
\hline ITO 100 & 335 & 372 & 394 & 430 & 1.28 \\
\hline $0.2 \% \mathrm{MF}$ & 5.25 & 5.43 & 5.52 & 5.6 & 1.06 \\
\hline $2 \% \mathrm{MF}$ & 0.83 & 0.76 & 0.77 & 0.77 & 0.92 \\
\hline
\end{tabular}

When the insulation resistance of transformer oil is higher than $100 \mathrm{G} \Omega$ and increases with time (Table 1, ITO 100) it can be used in transformer [8]. Otherwise, there can be some problems in the insulation stage. In our case the magnetic fluid was prepared from nanoparticles and new inhibited ITO 100 transformer oil. ITO 100 was without impurities. In this case, the decrease (Table 1) of the insulation resistance of magnetic fluid was caused by the presence of magnetic nanoparticles. From these results follows that this type of diagnostic method for determination of quality of insulation stage cannot be used for magnetic fluid based on transformer oil.

Development of arc currents in oil and magnetic fluids are presented in Fig 2. The arc current is characterized by under-damped oscillation with angular frequency $[9,10]$. The angular frequency is the function of electrode distance, type of liquid and outer parameters. As it can be seen from Fig. 2 the angular frequency is smaller for magnetic fluids. The angular frequency decreased with volume concentration of magnetic fluid. Amplitude and duration of arc current were changed with applied voltage at identical electrode distance and the magnetic fluid. The angular frequency did not changed with applied voltage. The measurements were also made at various electrode distances $(0.1-0.6 \mathrm{~mm})$ and similar developments and characteristics of arc currents were observed (Fig. 2)

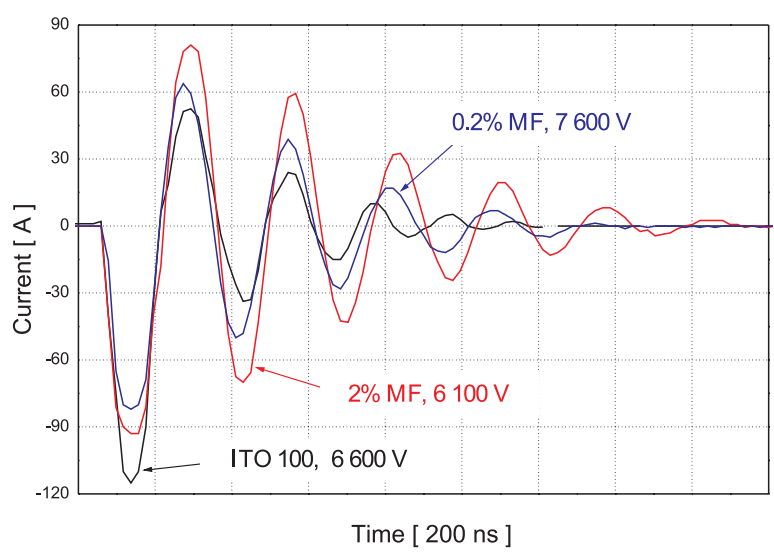

Fig. 2 The time dependence of arc current in transformer oil, $0.2 \%$ and $2 \%$ magnetic fluid with applied voltage (distance between the electrodes $d=0.4 \mathrm{~mm}$ ).

The breakdown voltage or dielectric strength is the main measured parameter for determination of quality of insulating fluids. Fig. 3 illustrates the dependencies of the DC breakdown voltage on the distance between the electrodes for transformer oil and two volume concentrations of magnetic field. The measurements in the case of magnetic liquid were performed also in the external magnetic field $(20 \mathrm{mT})$ in parallel orientation with the electric field (Fig. 1). Magnetic fluid was placed in the external magnetic fields during the whole measurement of the breakdown voltage.

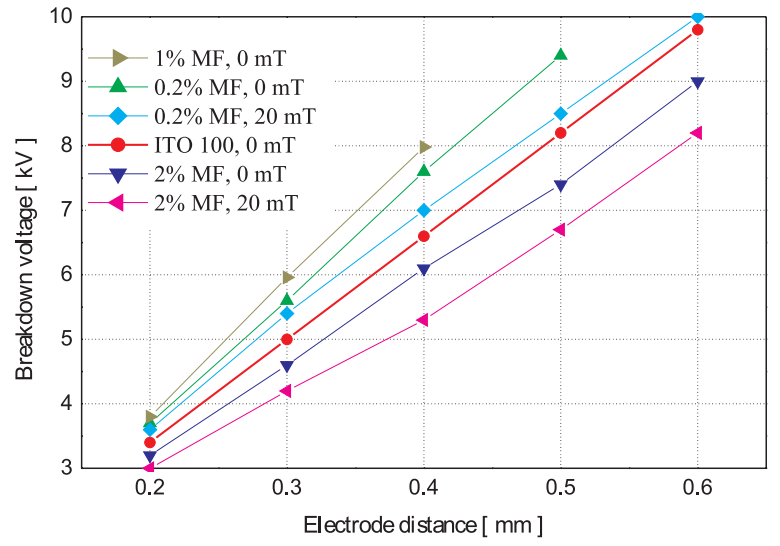

Fig. 3 The breakdown voltage versus distance between the electrodes for ITO 100 transformer oil and magnetic fluids (0.2\%, $1 \%$ and $2 \%)$ without the external magnetic field $(B=0 m T)$ and in the external magnetic field $(B=20 \mathrm{mT})$. 
The breakdown voltage of $0.2 \%$ and $1 \%$ magnetic fluid is higher than those of the transformer oil, even also in the magnetic field. These properties are worse in $2 \%$ magnetic fluid. This is in agreement with the observation for the DC impulse voltage of Segal [1] and Marton [2, 3] for both DC and AC voltage.

\section{Discussion}

The change of the breakdown voltage of magnetic fluid with different volume concentration can be interpreted using theory of breakdown transformer oil. This theory is based on the bubble mechanism of breakdown, which is supported by various experimental works [11-16]. The main aspect is formation of bubbles (small channels [9]), regions with smaller density than surrounding liquid. These bubbles are formed by local heating and fieldemitted electron injected in electrode space. By increasing voltage these regions grow and expand until their local density is reduced under a critical density [11] enough to take place in electron impact ionization. In the next phase, electron avalanches expand and create an ionizing front - primary streamer or streamers. When one of the streamers bridges the inter-electrode gap, plasma channel is generated and the breakdown can be observed. In the final stage of breakdown the energy stored in a capacitor is transformed to arc discharge and under damper oscillation of arc current can be observed $[9,10]$.

From previous we know that the value of breakdown voltage depends on creation of bubbles and processes running inside. When magnetic nanoparticles (e.g. magnetite $\mathrm{Fe}_{3} \mathrm{O}_{4}$ ) are added to transformer oil, there is negative effect on the formation of bubbles. The magnetite nanoparticles in transformer oil have extremely short charging time [16], so they are excellent electron traps. Trapped fast electrons are lost for ionization process and slow negative nanoparticles are produced. These negative ions also caused the decrease of electric field which had negative effect on ionization process. Nanoparticles are polarizable and have higher permittivity than the surrounding liquid, so they move by electrical force to the place of maximum of electrical intensity. This place is also the place of the creation and existence of bubbles. Therefore, when the formation and existence of bubbles are affected by magnetic nanoparticles, it results in the increase of breakdown voltage. This is observable only for small concentration of magnetic fluid, as in our case $0.2 \%$ and $1 \%$ (Fig. 3) [2, 3].
When magnetic fluid has higher volume concentration of magnetic particles ( $2 \%$, Fig. 3 ) we must take into account another effect. The magnetic nanoparticles are again moved to the place of maximum stress but their high concentration is presumed to be initiated by the surface irregularities on the electrodes and formations of the chains, which give rise to local field gradients. The accumulation of particles continues and tends to form a bridge across the gap, which leads to the breakdown initiation. In external magnetic field is the decrease of the breakdown voltage due to the magnetic dipol-dipol interaction between the particles and formations of the chains and chain-like elongated clusters [4, 5]

Water content is one of the main parameters, which can cause the decrease of the insulation resistance. Also small solid impurities and gases dissolved in oil have the same influence. In these experiments the decrease of insulation resistance was not caused by these impurities, because new ITO 100 transformer oil was used. The decrease of insulation resistance is uniquely determined by the presence of magnetic nanoparticles in these measurements. From these results it follows that when transformed oil contained magnetic nanoparticles the diagnostic method of the isolation resistances test cannot be used.

\section{Conclusion}

The insulating properties of magnetic fluid based on transformer oil with a small volume concentration $(0.2 \%$ and $1 \%)$ of magnetite nanoparticles were better in comparison with pure transformer oil. The presence of small concentration of magnetic nanoparticles has a profound effect on the breakdown voltage of liquid insulators. For $2 \%$ magnetic fluid the situation was opposite, the insulation properties were worse and breakdown voltage was also smaller. The change of breakdown voltage was interpreted on the base of the bubble theory of breakdown.

\section{Acknowledgements}

The authors wish to thank for the support to the R\&D operational program Centre of excellence of power electronics systems and materials for their components. The project is funded by the European Community, ERDF - European regional development fund.

\section{References}

[1] SEGAL, V., RAJ, K.: An Investigation of Power Transformer Cooling with Magnetic Fluids, Indian J. Eng. Mater. Sci. 5 (6), pp. 416-422, 1998,

[2] KOPCANSKY, P., TOMCO, L., MARTON, K., KONERACKA, M., TIMKO, M., POTOCOVA, I.: The DC Dielectric Breakdown Strength of Magnetic Fluids Based on Transformer Oil, Journal of Magnetism and Magnetic Materials, vol. 289, pp. 415-418, 2005.

[3] TOMCO, L., MARTON, K., HERCHL, F., KOPCANSKY, P., POTOCOVA, I., KONERACKA, M.: The DC and DC Insulating Properties of Magnetic Fluids Based on Transformer Oil, Phys. sta. sol (c) , 1/2006, pp. 195-198.

[4] JOZEFCZAK, A.: Study of Low Concentrated Ionic Ferrofluid Stability in Magnetic Field by Ultrasound Spectroscopy, Journal of Magnetism and Magnetic Materials, vol. 294, 2009 Article in Press, 
[5] BRAMANTYA, M. A., MOTOZAWA, M., TAKUMA, H., FAIZ, M., SAWADA, T.: Experimental Analysis of Clustering Structures in Magnetic and MR Fluids Using Ultrasound, Journal of Physics: Conference Series, vol. 149, 012040, 2009,

[6] KUFFEL, E., ZAEUNGL, W.S.: High Voltage Engineering Fundamentals, Pergamon Press, Oxford, 1984,

[7] GUTTEN, M., KORENCIAK, D.: Frequency Diagnostics Options of Transformers, In Diagnostika '09, Plzen 2009, pp. 78-81,

[8] http://www.megger.com/eu/index.php

[9] KUDELCIK, J.: Development of Breakdown in Transformer Oil, ADVANCES in Electrical and Electronic Engineering 6, pp. $35-39,2007$,

[10] KUDELCIK, J., GUTTEN, M.: Measurement of Electrical Parameters of Breakdown in Transformer Oil, In $7^{\text {th }}$ International Conference on Measurement, Smolenice 2009, pp. 393-396,

[11] JONES, H. M., KUNHARDT, E. E.: Development of Pulsed Dielectric Breakdown in Liquids, Journal of Physics D: Applied physics, vol. 28, pp. 178-188, 1995,

[12] DEVINS, J. C., RZAD J., SCHWABE, R. J.: Breakdown and Prebreakdown Phenomena in Liquids, Journal of Applied Physics, vol. 52, pp. 4531-4545, 1981,

[13] BEROUAL, A.: Electronic and Gaseous Processes in the Prebreakdown Phenomena of Dielectric Liquids, Journal of Applied Physics, vol. 73, pp. 4526-4533, 1993,

[14] TIMOSHKIN, V. et all: Hydrodynamic Modeling of Transient Cavities in Fluids Generated by High Voltage Spark Discharges, J. Phys. D.: Appl. Phys., vol. 39, pp. 4808-4817, 2006,

[15] KUDELCIKOVA, M.: Positive Solutions of Delayed Differential Equation, In Modelling \& Stability: international conference Dynamical system modelling and stability investigation, Kyjev, 2009, pp. 29-30.

[16] HWANG, J. G., O'SUlliVAN, F., ZAHN, M., HJORTSTAM, O., PETTERSON, L., LIU, R.: Modeling of Streamer Propagation in Transformer Oil-based Nanofluids, Annual Report - Conference on Electrical Insulation and Dielectric Phenomena, Article number 4772777, pp. 361-366, 2008. 